\title{
Towards Active Course Marks for Autonomous Sailing Competitions
}

\author{
Paulo Ferreira $^{1}$, Benedita Malheiro ${ }^{1,2}$, Pedro Guedes $^{1}$ and Manuel Silva ${ }^{1,2}$
}

\begin{abstract}
This paper describes an environmental monitoring / regatta beacon buoy under development at the Laboratory of Autonomous Systems (LSA) of Instituto Superior de Engenharia do Porto (ISEP). The buoy is a dual mode modular and configurable system that includes communications, control, data logging, sensing, storage and power subsystems. In environmental monitoring mode, the buoy collects and stores data from several underwater and above water sensors and, in regatta mode, the buoy becomes an active course mark as well as a data beacon for the autonomous sailing boats in the vicinity. During a race, the buoy broadcasts its position, together with the wind and the water current local conditions, allowing autonomous boats to navigate towards and round successfully the mark. This project started with the specification of the requirements of the dual mode operation, followed by the design and building of the buoy structure and is currently focused on the development of the modular, configurable, open source-based control system. The ultimate goal is that autonomous sailing regatta courses are defined using this type of active course marks, ensuring that the autonomous sailing boats can concentrate on the regatta strategy and tactics rather than on finding the marks.
\end{abstract}

\section{Introduction}

Monitoring and exploring maritime, river and lagoon environments is challenging for materials, electronic components and people. These vital ecosystems are under severe pressure due to human activities and businesses; therefore, agencies and governments need to deploy in situ versatile telemetry platforms to collect valuable data for assessment and sensible decision making.

Autonomous sailing and, in particular, the World Robotic Sailing Championship is a research competition that merges robotics and sailing with the aim to expand

(1) School of Engineering, Polytechnic Institute of Porto, Porto, Portugal

(2) INESC TEC - INESC Technology and Science, Porto, Portugal 
the frontiers of knowledge that supports the autonomous exploration of these environments. The competing autonomous boats are expected to sail a series of regattas following pre-defined courses to determine the winner team. In manned sailing competitions, the course, including the starting and finishing lines, as well as the course legs, is defined with the help of marks, usually large inflatable buoys, that all competitors must successfully round in the intended order and direction.

In order to address these two challenges, this paper proposes a platform that combines both environmental monitoring and regatta beacon modes. This dual mode platform is a reconfigurable environmental monitoring buoy that can be deployed to collect, $\log$ and communicate water and wind-related data as well as an active course mark, i.e., a regatta beacon, that broadcasts position, wind and water-related information relevant for the sailing boats in the vicinity. It can be classified as an autonomous surface floating moored buoy, with the ability to sense, store and communicate in batch mode. Furthermore, it is able to work as a single node or integrated in a multiple node system. Internally, it includes a distributed embedded system, hosting a master and a slave processor, an e-compass, an Inertial Measurement Unit (IMU), a Global Navigation Satellite System (GNSS) receiver, a Conductivity, Temperature, Depth (CTD) sensor package and a wind sensor as well as a Secure Digital (SD) card for data logging.

The main contributions of this proposal are: (i) dual mode operation - environmental monitoring and regatta beacon; (ii) distributed master-slave embedded architecture based on Open Source solutions; (iii) dynamical selection of the active sensor set and operation mode. The regatta beacon mode constitutes a novel application domain - the development of active course marks for sailing.

This paper is organised in five sections, namely the introduction, related work, functional requirements, buoy design and development and conclusions.

\section{Related Work}

The weather and the environmental conditions in maritime, river and lagoon ecosystems are typically monitored with the aid of buoys. The buoys act as remote sensing nodes or probes, which may also include storage and communication services, among other functionalities. They range from self-controlled or autonomous [7] [5] [8] to remotely operated, from moored, to drifting [4] or mobile [7] [8], from batch to real time communication [5] modes, from surface floating to underwater [7] [8] devices, from single node to multiple node systems [1] [7] and cover a wide spectra of application fields, e.g., pollution detection [6], hydrology system monitoring [2], shallow water ecosystem monitoring [1], bathymetric surveys [3] or underwater environmental monitoring [7].

The buoy station for marine pollutant detection proposed by [6] adopts spectroscopic and fluorescence methods for detecting specific pollutants. This instrumentation approach (spectroscopy) requires the monitoring system to be imune to vibrations, waves and shocks of the maritime environment. 
The low-cost multisensory buoy system proposed by [1] is intended for monitoring coastal shallow-water marine environments. The buoys have power autonomy as well as dimensions suitable for easy deployment and for the stability of the sensor system in an aggressive and dynamic environment like the sea. In terms of functionalities, the buoy system remotely monitors and records the temperature and marine pressure and the temperature and atmospheric pressure, i.e., can be integrated in a Wireless Sensor Network.

The GPS buoys created by [2] are equipped with high-quality geodetic GNSS receivers for stage monitoring of large rivers or lakes. In the specific case of hydraulic systems study, the buoys, which allow freedom of positioning and quick and easy deployment, provide a valuable tool that supplements the existing pool of hydrometric instruments by allowing water elevation and movement monitoring.

The autonomous buoy developed by [7] monitors the underwater environment with the help of an Optical Particle Counter (OPC) sensor and a CTD sensor package. The vehicle is an autonomous profiling float that uses a buoyancy engine to cycle horizontally while moving up and down. It is controlled by an embedded system composed of field-programmable gate array (FPGA) and high performance CPU, which is designed to perform image signal processing, data compression, power management and satellite communication.

Hidroboya ${ }^{\mathrm{TM}}$ is the autonomous buoy developed by [5] for real time water data retrieval. It includes a multi-parametric sensor that measures temperature, $\mathrm{pH}$, electrical conductivity, redox, turbidity and solubilized oxygen concentration. The buoy uses a hydraulic system to feed fresh ocean water to the sensors when required, keeping the sensors free from sea particles sedimentation. The water data is automatically sent, using a General Packet Radio Service (GPRS) data communication link, to a server for storage in a database. The server, which is capable of processing data from several buoys, allows authorized users to browse data over the Internet.

The Riverine Drifter is an autonomous river drifting buoy developed by QinetiQ North America (QNA) Technology Solutions. It floats freely in a river current and accurately collects and transmits, in real time, surface velocities, water depth and temperature as a function of position worldwide via satellite communications [4]. The riverine drifter includes a GPS, a depth sounder with temperature transducer and a dual-axis accelerometer, a Gumstix single-board computer running a Linux operating system, Iridium satellite and Bluetooth communications and a battery pack with an autonomy of $24 \mathrm{~h}$.

The Autonomous Mini-buoy designed by [8] is intended for use in a coordinated, wireless networked array of buoys for near-surface ocean sensing. These mini-buoys are mobile, they can keep stationary in currents as large as $25 \mathrm{~cm} / \mathrm{s}$ or move as an adaptive, coordinated sensor array for high resolution in both time and space. Deployment times are about 1 to 2 days. It includes a Proportional-IntegralDerivative controller with GPS and magnetic heading sensors to maintain course, a $900 \mathrm{MHz}$ communication system with a range of 1 to $2 \mathrm{~km}$ and plans for a longer range HF/VHF or satellite communication system and acoustic, wave, air and water temperature and GPS sensors. 
The Self-Positioning Smart Buoys proposed by [3] are used on oceanographic and bathymetric surveys. The ASC SCOUT model is based on a self-propelled, GPS-positioned, autonomous surface craft that can be pre-programmed, autonomous, or directed in real time. Each vessel can communicate wirelessly with deployment vessels and other similar buoys directly or via satellite. The ASC SCOUT vehicles deploy a CTD sensor package as well as acoustic modems, wave and wind measurement sensors and extended range communications hardware.

\section{Functional Requirements}

The buoy should act as an autonomous device commanded by the base station and offer two operation modes: environmental monitoring mode and regatta beacon mode. In environmental mode, the system should be able to receive request commands and transmit status or data via the wireless and Universal Serial Bus (USB) interfaces. The requests can be reconfiguration (include/exclude sensors, enabling/disabling an operation mode, etc.) or data retrieval related. In regatta mode, the buoy must, additionally, act as a beacon and broadcast via the wireless interface the Position, Velocity and Time (PVT), wind and water related data for autonomous boats located in the vicinity. If both modes are enabled, priority must be attributed to broadcast the information to the autonomous sailing boats.

The platform should be: (i) multi-purpose to optimize resources, i.e., the buoy operates both as an environmental monitoring device and as a regatta beacon, since the latter is a time limited activity; (ii) low-cost, i.e., the cost of the computational hardware, of the software development and of the mechanical design of the buoy needs to be minimized for the success of the buoy; (iii) modular and reconfigurable to support the inclusion/exclusion of real time broadcast, different types of sensors, and/or different types of monitoring tasks; (iv) reproducible so that the design employs as much as possible Open Source hardware and software components; ( $v$ ) easy to deploy. The global buoy design objectives are illustrated in Figure 1.

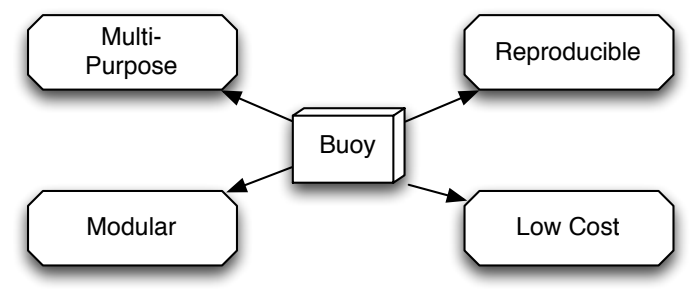

Fig. 1 Design Objectives 


\section{Buoy Design and Development}

The buoy design involved the conceptual design of the structure and of the control, sensing and communication architecture.

\subsection{Physical Structure}

The buoy is composed of a stainless steel structure, a fibreglass hull and a standard anchoring system. The hull is sealed in order to provide a waterproof environment and contains the electronic system. Figure 2 displays the hull, the design of the structure and the assembled set.
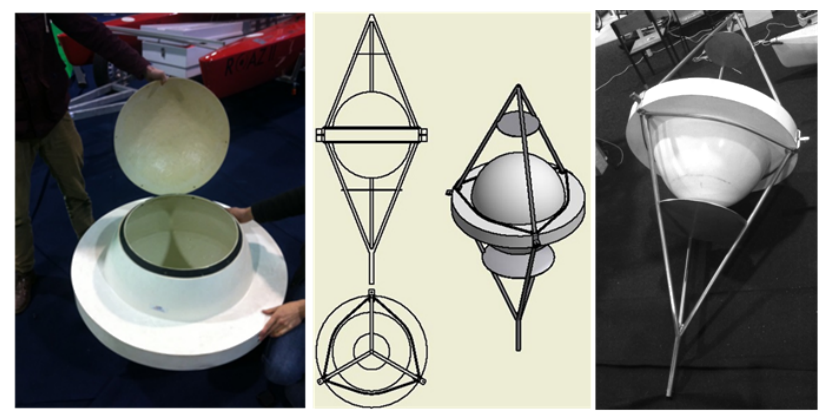

Fig. 2 Hull and stainless steel structure

The stainless steel structure is a frame that is attached to the hull ring in three places and protrudes upwards and downwards with three tubes. It consists of two almost symmetrical parts, divided in the horizontal plane, that are bolted together. In the upper part of the structure there is a horizontal, circular plate to which the wind and GNSS sensors, lamp and antennas can be attached. In the lower part there is a similar plate, but of a greater diameter to provide the space for the underwater CTD sensor. At the very bottom there is a cylindrical rod for the placement of circular weights as the ballast. Because these weights can have a mass ranging from 0.5 $\mathrm{kg}$ to $20 \mathrm{~kg}$, they can be easily arranged in such a way as to obtain a desired total weight accordingly to the existing needs. This design is beneficial in a couple of ways. Firstly, it allows easy access to the hull, and secondly, it is easier to combine both parts in the horizontal plane. At the bottom of the cylindrical rod there is a threaded hole for an eye bolt to which the anchor line can be directly attached. In order to make sure that the buoy stays in one place on a river, it needs to be connected to an anchor. However, the anchor is only one part of the overall anchoring system that is required for the buoy. This system consists of one anchor, two swivels, one shackle, one thimble, one nylon rope, one chain and one eye bolt. 


\subsection{Buoy Control Architecture}

The buoy control architecture presents a modular and distributed structure, with blocks responsible for underwater measurements, on-surface measurements, data collection and storage, communication and power supply (Figure 3). The Master Control Unit (MCU) receives the commands, process them and sends commands to the Slave Control Unit (SCU). In regatta mode it also broadcasts the navigation-aid information for autonomous boats. The slave executes several tasks regarding data collecting and data passing to the master. It also has the possibility of reconfiguring all sensors connected, switching them on/off, changing data sampling rates, etc.

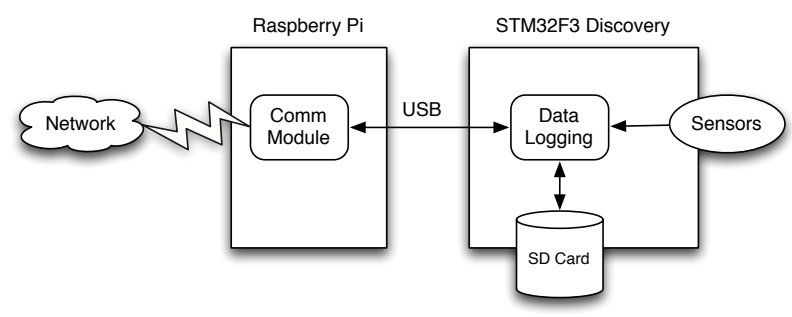

Fig. 3 Block diagram of the system implementation

For the MCU, an embedded Linux board was chosen. The decision was between the Raspberry Pi and BeagleBone Black. While the Raspberry Pi has more market share, the BeagleBone has more computational power and a wider range of connections. The choice has been left open and the buoy development is being done in a portable way so that any board (Raspberry Pi, BeagleBone Black or other) can be used on the final system. This is possible because the connection between the master and slave boards is USB-based. As a result, the proposed architecture is flexible, supports different types of boards, allows the interconnection of multiple data logging boards and uses a single networking board.

The measurements from the enabled sensors are collected and stored in the data storage device (SD card) and, then, are sent via the wireless interface on request to the base station and/or in real time to the boats. The data collection and logging is performed by the SCU - a STM32F3-Discovery board - and the communication and reconfiguration is done by the MCU - a Raspberry Pi board. The power supply is provided by $6 \mathrm{~V}$ and $12 \mathrm{~V}$ batteries.

\subsection{Telemetry}

The main function of the buoy is to do telemetry, i.e., to autonomously measure and store parameters in situ and to send them, on request (environmental monitoring) 
or continuously (regatta beacon), via a wireless link to the receiving equipment. In the current stage of the project, the following parameters are contemplated: (i) wind speed and direction; (ii) water conductivity, temperature and depth; (iii) PVT; (iv) heading; and $(v)$ acceleration, pitch, roll and yaw. In the near future, the water current direction and velocity are to be included. Figure 4 displays a block diagram of the MCU. The MCU is the networking module since it provides point-to-point communication between the base station and the buoy, point-to-multipoint communication between the buoy and the boats, and the internal communication with the slave unit.

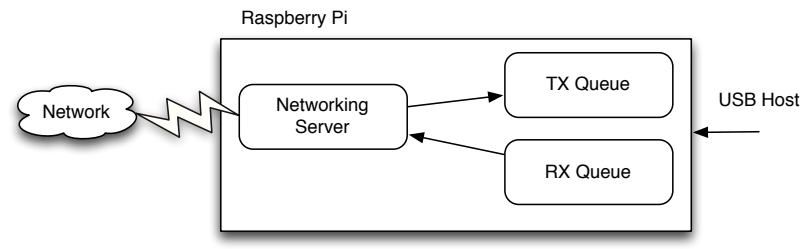

Fig. 4 Networking module

Furthermore, the MCU allows the base station to reconfigure the buoy remotely. This reconfiguration corresponds to the selection of the operation mode and of the list of enabled sensors. The system, in environmental mode, receives request commands and transmits status or data via the wireless and USB interfaces. In regatta mode, the buoy, additionally, acts as a beacon that broadcasts the PVT, wind and water related data for the autonomous boats located in the vicinity.

\subsection{Sensors and Data Logging}

The list of microcontroller candidates for sensor interfacing, data acquisition and logging is large. The STM32F3 Discovery Kit was chosen because it has a low price (less than $10 €$ ), a $72 \mathrm{MHz} 32$ bit processor with $256 \mathrm{KiB}$ of Flash memory, $48 \mathrm{KiB}$ of RAM and has a built-in USB interface to interconnect with the Linux board. Besides the processor, the board also includes a 3D digital output gyroscope, a 3D digital linear acceleration sensor and a 3D digital magnetic sensor. The board has another USB interface that works as an ST-LINK debug interface and that can be used with a common Open Source embedded debug tool like the Open On-Chip Debugger (OpenOCD) / GNU Project debugger (GDB) pair. The processor/board combination is supported by GCC and by various Open Source Real-Time Operating Systems like ChibiOS1 The GCC/GDB/ChibiOS association allows an Open Source portable development environment (runs on Windows/Linux/OSX), offers support for threads, semaphores, mutexes, messages, queues and includes device drivers for

$1 \longdiv { \text { http://www.chibios.org } }$ 
the board's peripherals. ChibiOS was chosen because it offers the widest support, not only regarding different development hosts, but also many device drivers for a wide range of peripherals.

The STM32F3-Discovery board performs, as a SCU, the following tasks: (i) continuously collects and stores the measurements from the above water, underwater and its internal sensors; and (ii) receives reconfiguration commands from the MCU. Figure 5 displays the data logging and sensor interface module.

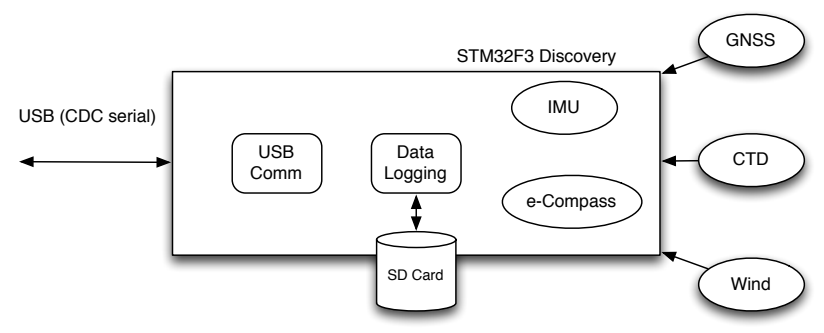

Fig. 5 Data logging and sensor interface module

\subsection{Communication Protocol}

The communication between the base station and the MCU as well as between the MCU and SCU adopts a binary protocol. This architecture isolates intentionally the base station from the slave module. Figure 6 shows the message data format. Furthermore, Table 1 and Table 2 complement this description.

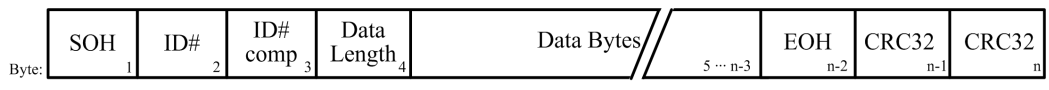

Fig. 6 Message data package

The MCU processes several commands simultaneously and uses different threads to receive and send data from/to the base station and from/to the slave. The commands, which have different priorities, are processed accordingly by the master by implementing data queues. The commands are grouped as shown in Table 2 
Table 1 Description of byte data package

\begin{tabular}{|c|c|c|}
\hline Length (B) & Abbreviation & Function \\
\hline 1 & $\mathrm{SOH}$ & The '\$' sign is used as Start Of Header. \\
\hline 2 & ID\# & $\begin{array}{l}\text { Number that represents the identification of message } \\
\text { (commands or errors). }\end{array}$ \\
\hline 3 & ID\# Complement & $\begin{array}{l}\text { First complement of the ID number. This is used as a } \\
\text { validation of the ID. }\end{array}$ \\
\hline 4 & Data Length & Number that indicates the size in $B$. \\
\hline $5, n-3$ & Data Bytes & Actual data (in accordance with ID\#) that is sent. \\
\hline$n-1$ & $\mathrm{EOH}$ & The '*' is used as End Of Header. \\
\hline$n-1, n$ & CHC32 & Checksum: $16 \mathrm{~b}$ sum of the entire package. \\
\hline
\end{tabular}

Table 2 ID of messages - commands and errors

\begin{tabular}{clclc}
\hline ID\#(1st digit) & Function & ID\#(2nd digit) & Parameter & Priority level \\
\hline 1 & Mode selection & 0 & On & 3 \\
& & 4 & Off & 5 \\
\hline 2 & GPS & 3 & Coordinates & 6 \\
\hline 3 & Wind & 1 & Wind speed & 7 \\
& & 6 & Wind direction & \\
\hline 4 & Water & 1 & Water depth & 4 \\
& & 5 & Water conductivity & 1 \\
\hline \multirow{2}{*}{6} & Operation & 9 & Water temperature & 8 \\
& & 2 & Combination & \\
\hline 7 & Sensor & 7 & Stop & 2 \\
& & 1 & On & \\
\hline 8 & Error & 6 & Off & \\
\hline & & 2 & SDC NOK & NA \\
\hline
\end{tabular}

\section{Conclusions}

This paper presents the design and development of a dual mode buoy under development at ISEP. The main contributions of this proposal are: $(i)$ dual mode operation environmental monitoring and regatta beacon; (ii) distributed master-slave embedded architecture based on Open Source solutions; (iii) dynamical selection of the active sensor set and operation mode. The regatta beacon mode constitutes a novel application domain - the development of active course marks for sailing. 


\subsection{Discussion}

The selection of a Linux general purpose embedded board for the MCU allowed the use of the Linux networking facilities to support the communications subsystem and the USB interface to connect with the SCU straightforwardly. The programming of this module was done in Python since it has a good performance and a rapid development cycle. Moreover, it can easily reuse routines written in $\mathrm{C}$, sidestepping any eventual processing speed limitations.

The adoption of the STM32F3-Discovery board for the data logging and sensor interface module provided a good performance versus power consumption relationship, relevant on board sensors and an extensive set of interfaces. Additionally, it allowed the installation of ChibiOs as a real time operative system, offering high level drivers for the peripherals and a vast set of communication and synchronization primitives on a multithreading programming model.

The set of sensors contemplated can be easily upgraded and extended, e.g., the inclusion of sensors for measuring the water current direction and velocity.

\subsection{Future Developments}

In the near future, "field" tests will be performed to evaluate and debug the platform.

In order to make the buoy fully autonomous, renewable energy sources, e.g., a solar panel, wind turbine or water current generator, can be adopted. Another possibility is the development of an energy harvesting device, in order to provide electrical power to the buoy, taking advantage of the ripple of the waves.

In terms of software applications, the development of a portable software client for easy inclusion on autonomous boats and a robust base station server is planned.

For environmental monitoring purposes, the evaluation of special networking modes (Ad-Hoc) and the possible power control of the communications module by

the sensor module is foreseen. This will allow the automatic data transfer between a set of buoys, and provide a longer battery life.

Acknowledgements The authors thank Bennet Moller, Emil Wlazlo, Mateusz Tarkowski, Toon Van den Bleeken, Hendrik Verschelde, Jeroen Vervenne, Laurens Allart, Mathias van Flieberge for their work on this project, António F. Silva, Maria Cristina Ribeiro, Nídia Cetano, João Paulo Baptista and Fernando Ferreira for their expertise, ALTO - Perfis Pultrudidos, Lda for the fibreglass hull and, finally, to the LSA at ISEP. This work was partially supported by the ERDF - European Regional Development Fund through the COMPETE Programme (operational programme for competitiveness) and by National Funds through the FCT - Fundação para a Ciência e a Tecnologia (Portuguese Foundation for Science and Technology) within project "FCOMP - 01-0124FEDER-022701". 


\section{References}

[1] Albaladejo, C., F. Soto, R. Torres, P. Sánchez, and J. A. López (2012). A low-cost sensor buoy system for monitoring shallow marine environments. Sensors 12(7), 9613-9634.

[2] Apel, H., N. Hung, H. Thoss, and T. Schüne (2012). GPS buoys for stage monitoring of large rivers. Journal of Hydrology 412-413(0), 182-192. Hydrology Conference 2010.

[3] Curcio, J., P. McGillivary, K. Fall, A. Maffei, K. Schwehr, B. Twiggs, C. Kitts, and P. Ballou (2006, Sept). Self-positioning smart buoys, the "un-buoy" solution: Logistic considerations using autonomous surface craft technology and improved communications infrastructure. In OCEANS 2006, pp. 1-5.

[4] Emery, L., R. Smith, R. McQuary, B. Hughes, and D. Taylor (2011, Sept). Autonomous river drifting buoys - applications and improvements. In OCEANS 2011, pp. 1-5.

[5] Fernandez-Hermida, X., C. Duran-Neira, M. Lago-Reguera, C. RodriguezAlemparte, and F. Martin-Rodriguez (2011, June). Hidroboya: An autonomous buoy for real time high quality sea and continental water data retrieval. In OCEANS, 2011 IEEE - Spain, pp. 1-7.

[6] Griffo, G., L. Piper, A. Lay-Ekuakille, and D. Pellican (2014). Design of buoy station for marine pollutant detection. Measurement 47(0), 1024-1029.

[7] Lee, K. W., D. Lee, U. Jeong, J. Y. Yang, H. K. Jun, and J.-H. Park (2011, June). Implementation of embedded system for autonomous buoy. In OCEANS, 2011 IEEE - Spain, pp. 1-4.

[8] Vesecky, J., K. Laws, S. Petersen, C. Bazeghi, and D. Wiberg (2007, July). Prototype autonomous mini-buoy for use in a wireless networked, ocean surface sensor array. In Geoscience and Remote Sensing Symposium, 2007. IGARSS 2007. IEEE International, pp. 4987-4990. 